\title{
The impact of the mode of thought in complex decisions: intuitive decisions are better
}

\author{
Marius Usher, 2* , Zohar Russo', Mark Weyers' ${ }^{3}$, Ran Brauner ${ }^{1}$ and Dan Zakay ${ }^{1,4}$ \\ 1 Department of Psychology, Tel-Aviv University, Tel-Aviv, Israel \\ 2 School of Psychological Science, Birkbeck College, University of London, London, UK \\ ${ }^{3}$ University College London Centre for the Advancement of Learning and Teaching, University College London, London, UK \\ ${ }^{4}$ Interdisciplinary Center, Herzliya, Israel
}

Edited by:

Norbert M. Seel, University of Freiburg,

Germany

\section{Reviewed by:}

Richard J. Tunney, University of

Nottingham, UK

Christoph T. Weidemann, Swansea

University, UK

*Correspondence:

Marius Usher, Department of

Psychology, Tel-Aviv University, Ramat

Aviv, Tel-Aviv 69978, Israel.

e-mail:marius@post.tau.ac.il
A number of recent studies have reported that decision quality is enhanced under conditions of inattention or distraction (unconscious thought; Dijksterhuis, 2004; Dijksterhuis and Nordgren, 2006; Dijksterhuis et al., 2006). These reports have generated considerable controversy, for both experimental (problems of replication) and theoretical reasons (interpretation). Here we report the results of four experiments. The first experiment replicates the unconscious thought effect, under conditions that validate and control the subjective criterion of decision quality. The second and third experiments examine the impact of a mode of thought manipulation (without distraction) on decision quality in immediate decisions. Here we find that intuitive or affective manipulations improve decision quality compared to analytic/deliberation manipulations. The fourth experiment combines the two methods (distraction and mode of thought manipulations) and demonstrates enhanced decision quality, in a situation that attempts to preserve ecological validity. The results are interpreted within a framework that is based on two interacting subsystems of decisionmaking: an affective/intuition based system and an analytic/deliberation system.

Keywords: complex decisions, intuition, affect, distraction, implicit learning, two-system theory

\section{INTRODUCTION}

Research in the last 20 years indicates that human cognition involves two qualitatively different processing modes. The first one is fast, affective, parallel, associative, and holistic and has been attributed to the operation of an experiential/affective or intuitive system, while the second is slower, sequential, rule-based, and analytic and has been attributed to the operation of a rational or a deliberation system (Epstein, 1994; Sloman, 1996; Evans, 2003, 2008; Kahneman, 2003; Ferreira et al., 2006; Kahneman and Frederick, 2007; but see Keren and Schul, 2009). As each of these processing modes has its strengths and limitations, for each cognitive activity, optimal performance requires an adequate mix of the two modes. Decision-making is a cognitive activity, which has traditionally been thought of as the normative play-field of rational deliberation. Accordingly, one should consider the various aspects of the alternatives, consciously evaluate the risks and values, and decide through careful analysis the expected (or weighted) utility of each option. This prescription, which is part of the normative decision approach (subjective expected utility; Savage, 1954, or multiattribute weighted utility; Dawes and Corrigan, 1974; Keeney and Raiffa, 1976; Edwards and Fasolo, 2001; Edwards and Newman, 2003) is illustrated by the famous decision advice given by Benjamin Franklin to his friend Joseph Priestly: "When these difficult cases occur, they are difficult, chiefly because, while we have them under consideration, all the reasons pro and con are not present to the mind at the same time... To get over this, my way is to divide half a sheet of paper by a line into two columns; writing over the one pro, and over the other con; then during three or four days' consideration, I put down under the heads short hints of the different motives that at different times occur to me, for or against the measure. When I have thus got them all together in one view, I endeavor to estimate their respective weights; and, where I find two (one on each side) that seem equal, I strike them both out. If I find a reason pro equal to some two reasons con, I strike out the three. If I judge some two reasons con, equal to some three reasons pro, I strike out the five; and thus proceeding I find at length where the balance lies; and... I come to a determination accordingly." (Franklin, 1772).

Initial research in decision-making has provided support for this view, in the form of a variety of decision biases that have been attributed to the shortcomings of the intuitive mode (Kahneman, 2003). Prominent examples are reference and decoy effects, lossaversion, and violations of invariance and of transitivity, which have been explained by assuming that intuitive decisions involve the deployment of a set of heuristics (Tversky, 1969; Tversky and Kahneman, 1974; Gigerenzer and Goldstein 1996). Similarly, affect has also been associated with decision biases thought to reflect a type of heuristic (Winkielman et al., 1997; Raghunathan and Pham, 1999; Finucane et al., 2000; Au et al., 2003). Recent work, however, indicates a need to re-evaluate this assessment. First, it has been proposed that the association between heuristics (such as lexicographic rules, responsible for violations of intransitivity; Tversky, 1969) and intuition in decision-making is unwarranted. In a series of studies, Glöckner and colleagues have demonstrated that one can distinguish experimentally, between the deployment of such heuristics, which are sequential and rule-based, and the deployment of fast/parallel but compensatory modes of decisionmaking, which characterize the intuitive mode (Glöckner, 2008; Glöckner and Betsch, 2008a,b; see also Holyoak and Simon, 1999, for previous work on decision-making by constraint-satisfaction). Furthermore, recent studies have reported that framing effects (e.g., 
in the Asian disease problem) increase (rather than decrease, as the traditional view predicts) with the motivation to deliberate (Igou and Bless, 2007). Second, it is possible that the traditional view has underestimated the power of the intuitive/affective mode. Herbert Simon, who was the first to appreciate the capacity limitations of conscious processing in decision-making, has warned us that: “... there is a complete lack of evidence that, in actual choice situations of any complexity, these [rational] computations can be, or are in fact, performed... but we cannot, of course, rule out the possibility that the un-conscious is a better decision maker than the conscious." (Simon, 1955, p. 104).

Simon's warning is now supported by two lines of research. First, neuropsychological studies of decision-making and of social cognition have indicated the existence of an affective or somatic process, which under certain conditions plays a substantial (rather than a biasing) role in value integration and in intuitive judgments (Bechara et al., 1994; Damasio, 1994; Lieberman, 2000; Stout et al., 2004; Bechara and Damasio, 2005; Fellows, 2006; Weller et al., 2007; for review see Weber and Johnson, 2009). For example, affect is thought to provide a common currency, which is essential for value integration (Weber and Johnson, 2009), while intuition can be characterized as "the subjective experience of a mostly non-conscious process that is fast, a-logical and inaccessible to consciousness that, dependent on exposure to the domain or problem space, is capable of accurately extracting probabilistic contingencies" (Lieberman (2000, p. 111). This process has a different (holistic) nature, and can be distinguished experimentally, from the rule-based, sequential decisions that are subject to capacity limitations in working memory (Glöckner, 2008; Glöckner and Betsch, 2008a,b). Second, experimental studies have indicated that conscious deliberation (explicitly analyzing reasons when making decisions) can have a disruptive effect on attitudes (Wilson and Schooler, 1991; Wilson et al., 1993, 1995) and that distraction - a process likely to reduce the contribution of the deliberation process in decision-makingis not always detrimental for product evaluation (Betsch et al., 2001). For example, Betsch et al. (2001) showed that participants (who are simultaneously engaged in a distracting task) are able to differentiate good from poor shares on the basis of 75-150 numerical return values that were presented at a fast speed. Notably, this differentiation was possible only when the participants were probed using an affective cue (e.g., which share feels better) rather than when they were probed using an analytic one (report which of the shares had the highest average).

More recently, the hypothesis that the processes associated with intuition can facilitate decision quality has been supported by a series of studies by Dijksterhuis and colleagues, who proposed that, in complex decisions, decision quality is enhanced by setting the problem aside (from conscious scrutiny) after the initial (conscious) encoding, or in other words, by "sleeping on it" (Dijksterhuis, 2004; Dijksterhuis and Nordgren, 2006; Dijksterhuis et al., 2006; Bos et al., 2008; Strick et al., 2010). To show this they developed a decision-making paradigm that allows estimating an objective (but see discussion below) measure of decision quality and probing the impact that factors associated with intuition have on decision-making. The paradigm involves a contrast between a distraction and a "think-carefully" manipulation, on participants faced with a decision between three and four choice alternatives, each characterized by the same set of 10 or 12 binary attributes. For example, participants are asked to make a decision about their preferred choice of four cars based on a set of positive and negative attributes (such as good/poor mileage, good/poor handling, etc.). One alternative contains a majority $(66 \%)$ of positive attributes, another contains a majority $(66 \%)$ of negative attributes, while the other two alternatives lie in between (50\% positive/negative). Before the information is presented, the participants are required to form an impression about the various options. After all the information has been presented the participants are separated into two groups. The deliberation (or "thinkcarefully") group is asked to think-carefully for a predetermined interval of time (3-7 min) about which of the cars they prefer, based on the information they have been given. The distraction group is also asked to make a decision about their preferred choice of car, however they are first asked to complete an anagram task (of the same duration as the "think" period of the deliberation group), whose aim is to distract the participants from conscious deliberation. Sometimes a third group, an immediate decision group is also used as a baseline.

The surprising result (from the traditional theory viewpoint; e.g., Franklin, 1772; Edwards and Fasolo, 2001; Edwards and Newman, 2003) is that decision-making is enhanced in the distraction group, compared to the deliberation group (or to the immediate one; Dijksterhuis, 2004; Dijksterhuis et al., 2006). This result, often described as the unconscious thought effect, was then interpreted to support the unconscious thought theory (UTT), which claims that unconscious incubation processes during the distraction interval are responsible for the enhanced performance compared to the conscious deliberation, which is subject to severe capacity limitations (Dijksterhuis and Nordgren, 2006). UTT is related to the intuition-mode hypothesis, because one can assume that decisions performed in the distraction condition rely to a higher degree on intuitive strategies, than decisions performed after deliberation. Furthermore it is possible that immediate decisions fall in between, with participants in this condition using a mix of intuitive and deliberation strategies. One difference between these views is that UTT further assumes the presence of goaldirected unconscious thought during incubation (Dijksterhuis and Nordgren, 2006).

These results, however, have provoked vigorous debate, which centers on both conceptual criticism and on issues of replication. Examples of conceptual criticism involve the objective measure for decision quality (is it really the case that one option with $66 \%$ positive attributes is necessarily better for the participant than an option with 33\%? Gonzalez-Vallejo et al., 2008); it is thus important to ensure that the "best" alternative is defined relative to the subjective preference of the participant (as reflected by her ranking of the importance of the various attributes for the choice), and not only relative to the number of positive attributes. Further, there have been criticisms that center on the interpretation of the results: are unconscious decisions enhanced, or are the effects just a byproduct of interference triggered by the enforced deliberation with inadequate memory? (Shanks, 2006; Payne et al., 2008; Lassiter et al., 2009). Consequently, it has been argued that the results are an artifact of setting up participants to make deliberations in a situation that is of low ecological validity and of little 
relevance to real-life decision-making (Shanks, 2006; Lassiter et al., 2009). Further, replication problems were reported in a number of studies, which employed very similar methods and materials (Acker, 2008; Calvillo and Penaloza, 2009; Newell et al., 2009; Rey et al., 2009; Thorsteinson and Withrow, 2009). A number of studies by researchers associated with other labs, however, have reported replications (Lerouge, 2009; Lassiter et al., 2009; see discussion below) as well as generalizations to other domains (Ham et al., 2009; Ham and Van den Bos, 2010). Two recent meta-analyses have reported mean effect sizes of $g=0.251$ in favor of unconscious thought when compared to conscious thought (Acker, 2008) and $g=0.402$ in favor of unconscious thought when compared to both conscious thought and immediate judgments and decisions (Strick et al., unpublished manuscript ${ }^{1}$ ).

These diverging results indicate that the unconscious thought effect depends on subtle procedural differences and is subject to individual differences (Lassiter et al., 2009). Two new studies provide some possible explanations for the variability in results. First, Strick et al. (2010) have shown that the unconscious thought effect only takes place for participants that did not make their decision during the presentation of the material (online decisions; see also Lassiter et al., 2009), but rather postponed their decision for after the distraction manipulation (see further discussion after Experiment 1). Second, one of the most revealing studies using this paradigm (Lerouge, 2009), indicated that the advantage conferred by the distraction only impacts decision-making when the participants are induced into a holistic or configural mind-set (they are asked to form a global impression of each alternative) as opposed to a featural one (they are asked to compare individual attributes of the alternatives). This is important as holistic processing is directly linked to the intuitive/affective system, which is thought to mediate these effects.

The aim of the present work is to further investigate the impact of the mind-set and of distraction manipulations on the quality of decision-making. Our working hypothesis is that the unconscious thought effect is the outcome of the processing advantage of the intuitive/affective mode of thought (over the conscious, capacity limited, mode) in value integration. Accordingly, distraction is only one type of manipulation that can engage the intuitive/affective mode. In particular, when faced with complex multi-attribute decisions, participants can either rely on the power of the intuitive system to integrate values - an ability that is consistent with a vast amount of literature in implicit learning (Reber, 1993; Knowlton et al., 1996; Lieberman, 2000; Bierman et al., 2005) - or rely on a conscious system, which bases the decision on the few attributes that are maintained in working memory. This hypothesis shares many common principles with the UTT theory (for example the recommendation that better decisions are obtained, if ones "sleeps on it"), but does not need to subscribe to all the assumptions made about unconscious thought in UTT (Dijksterhuis and Nordgren, 2006).

Here we report the results of four experiments. The first one is a replication of the unconscious thought effect, with the attempt to ensure the validity of the subjective decision criterion. We believe this is important in light of the reported failed replications and of criticisms of the validity of the subjective decision criterion (Gonzalez-Vallejo et al., 2008). The second and third experiment,

${ }^{1}$ www.unconsciouslab.com/publications/Paper_Meta.doc examine the impact of mode of thought manipulations on decisionmaking, without a distraction manipulation. Here we reasoned that if the intuitive mode results in better decisions compared to the deliberation mode, we should be able to observe this by comparing the decision quality of deliberation vs. intuitive/affect manipulations in immediate decisions (with no distraction). Finally, in the fourth experiment we combine these two methods, by using a mode of thought in conjunction with a distraction manipulation. This combination is aimed to engage the intuitive system in a more efficient way. Further, we pose the following question (Experiments 3 and 4): Can we show that the intuitive/affective mode of thought improves decision quality in a situation that attempts to enhance the ecological validity of the decision?

\section{EXPERIMENT 1: A REPLICATION OF THE UNCONSCIOUS THOUGHT EFFECT}

The experiment used the unconscious thought paradigm with one important modification. Before being presented with the information about the four choice alternatives, each participant ranked the set of attributes on which the alternative choices were composed. These ranks were then used, via a computer program, to create alternatives for each participant, that ensured one of the alternatives (labeled here as A) was the best option for that participant; while another alternative (D) was the worst option for that participant (the other two options fell in between).

\section{METHOD \\ Participants}

Fifty-two participants (33 female) took part in this experiment. Twenty-four of them were from Birkbeck College (London; agerange 20-50) and 28 from Tel-Aviv University (TAU; age-range 20-30). The Birkbeck participants were tested in English while the Tel-Aviv participants were tested in Hebrew (the same materials were used and translated into Hebrew). In both groups, the participants were randomly assigned to the think-carefully $(N=27)$ or the distraction $(N=25)$ conditions, and to a car or holiday problem (see below). They were paid for their participation ( $£ 5$ or NIS25, respectively).

\section{Materials}

The choice materials were either four cars or four holiday packages, composed of 12 attributes each. The car attributes were taken from Dijksterhuis et al. (2006), and the holiday attributes were designed for this experiment (see Appendix). For both of the choice materials, the way in which the alternatives were constructed is illustrated in Table 1.

Table 1 | Positive and negative attributes of alternatives in Experiment 1.

\begin{tabular}{llll}
\hline Alternative & $\begin{array}{l}\text { No. of positive } \\
\text { attributes }\end{array}$ & $\begin{array}{l}\text { No. negative } \\
\text { attributes }\end{array}$ & $\begin{array}{l}\text { Rank of positive } \\
\text { attributes }\end{array}$ \\
\hline A & 8 & 4 & $1-8$ \\
B & 8 & 4 & $1,3-4,6-7,9-10,12$ \\
C & 6 & 6 & $1-6$ \\
D & 5 & 7 & $2,5,7,8,11$
\end{tabular}


Thus option-A had 8 (out of 12) positive attributes, which were the attributes ranked by the participant as the most important to them. Option-B also had eight positive attributes, but these attributes had lower rankings. Option-C had only six positive attributes but these attributes, similar to A, were the highest ranking attributes. The worst option was $\mathrm{D}$, which had only five positive attributes, and those were also attributes with lower rankings, compared to A (see Table 1). In the following we consider option-A as the best, options B and C (as middle options; we have no reason to predict that one is better than the other), and option-D as the worse.

\section{Procedure}

Stage-I. The experiment was described as an experiment on decision-making. Participants were asked to rank the various attributes of a car (or holiday package) in order of importance to them. The ranks were solicited by a computer program, which also registered them and used them online to construct the material for Stage-II. For example, a participant could indicate that the most important attribute for a holiday package is the "weather-forecast," and the second one, the "quality of beaches," etc. (see Appendix for the List of Attributes).

Stage-II. Participants were asked to form an overall impression about the four cars/holiday packages and were then presented with information about the alternatives. The order was randomized with regards to all combinations of alternative and attributes (Dijksterhuis et al., 2006) and the pace of presentation was 4 s per item (car and attribute combination). The alternatives were created as described above on the basis of the ranks that the participant gave in Stage-I. For example, for a participant who ranked "weather" as the most important attribute for a holiday package, the good option (A) was characterized by "good weather," as well as by positive attributes on the eight attributes the participant ranked highest; while the bad option (D) was characterized by "bad weather," and by negative attributes on six other attributes (see Table 1). The actual label (A-D), in which the four alternatives were presented to each participant was also randomized by the software.

Stage-III. Participants were divided into Think and Distraction groups. The think-group participants were told that they have to "think-carefully" for 3 min about which option they think is the best based on the information they were presented with. In contrast, the distraction group participants were asked to solve anagrams of animals for $3 \mathrm{~min}$, prior to deciding on their preferred option. The anagrams (words with a rearranged order of letters, e.g., dbri for target "bird") were presented on paper.

Stage-IV.All participants were asked to enter a rating for each of the four alternatives on a scale (1-10). The participants were instructed to first decide, which of the alternatives they prefer most and to give the highest rating to that alternative. Thus one could not enter equal ratings for the two most preferred alternatives.

\section{Dependent variables and statistical tests}

The dependent variables are the number of participants that rated the "good" alternative (A) highest (labeled here as "choice") and the ratings given to the best, middle and the worst alterna- tives. One variable that can be extracted from these ratings is the differentiation, computed as the rating difference: A-D. The UTT hypothesis predicts that the number of participants choosing the "best" option (A) will be higher for the distraction group. Second, the UTT hypothesis predicts an interaction in the rating between the groups and value given to the best, middle, and worse alternative, so that the distraction group should have a higher differentiation. Despite these predictions, we use here two-tail tests of significance, in order to maintain a cautious interpretation, in light of failed replication reports (Acker, 2008).

\section{RESULTS}

As there were no differences (in either choice or differentiation scores) between the participants who performed the task with cars and those who performed it with holidays, the results are collapsed across the two types of material.

\section{Choice}

The number of participants rating each option highest, in each of the two groups, is reported in Table 2. The fraction of participants who "choose" option-A (rather than any other option) was higher in the distraction group, compared with the think-carefully group $\left(\chi^{2}(1)=7.65, p<0.01\right)$.

\section{Differentiation}

The mean evaluations of the two groups for each of the four alternatives are shown in Figure 1.

We subjected this data to a 2 (group) $\times 3$ [quality: $A,(B+C) / 2$, D], ANOVA. The results indicated a highly significant main effect of quality $F(1,50)=42.3, p<0.001$, and an interaction between group and quality, which approaches, but does not reach significance $[F(1,50)=3.6 ; p=0.06]$. Participants gave higher ratings to

Table 2 | Number of participants rating each option highest, in each of the two groups.

\begin{tabular}{llllll}
\hline Group\alternative & A & B & C & D & $\begin{array}{l}\text { Total } \\
\text { participants }\end{array}$ \\
\hline Think-carefully & 8 & 10 & 7 & 2 & 27 \\
Distraction & 17 & 5 & 3 & 0 & 25
\end{tabular}

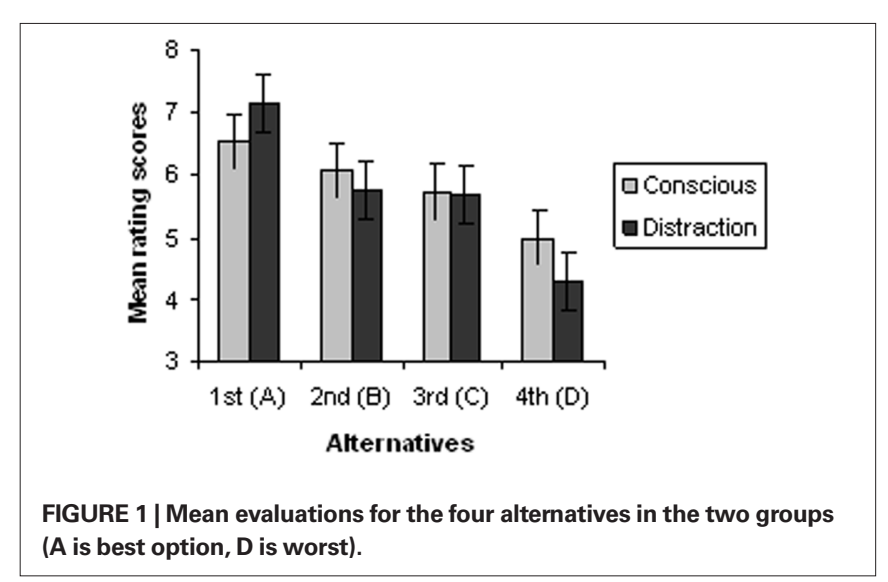


the better options, indicating that the ratings reflect the subjective importance of the attributes (entered by the participants) and the composition of the alternatives (Table 1). Furthermore, this discrimination tended to be larger for the distraction group; the A-D evaluation of the distraction group was (Mean $=2.96, \mathrm{SD}=2.64$ ), while that of the think-carefully group was [Mean $=1.62 ; \mathrm{SD}=2.46$; $t(50)=2.42, p=0.06]$.

\section{DISCUSSION}

While the ratings of the alternatives showed only marginally significant group differences in favor of the distractor group, the fraction of choices in favor of the best-alternative was highly significant, consistent with the UTT hypothesis (Dijksterhuis, 2004; Dijksterhuis et al., 2006). One possible criticism of this interpretation is that the participants in the distraction group avoided the badalternative D, because they made use of a lexicographic strategy choosing on the basis of the most important attribute - which puts $\mathrm{D}$ at a disadvantage (it has a low value on the most important attribute). This interpretation, however, is unlikely, in light of the choice results in Table 2, which show that only two of the participants (in the think-carefully group), actually chose the poor optionD. Eliminating these two participants from the analysis (focusing thus on choices of A vs. B and $\mathrm{C}$ ), shows that the advantage of the distraction group remains significant $\left[\chi^{2}(1)=6.48, p<0.02\right]$. One novel feature of this experiment is the fact that, although the alternatives were not arranged in a strict domination relation (one option better than another on a subset of attributes and equal to it in all the others, and thus one cannot simplify the problem by ignoring the common attributes), they were such that, given the ranking of the attributes by each participant, option-A was best and option-D was the worst for that participant. Moreover, this subjective order of preference is not subject to a psychological dissonance criticism: the distortion of the ranking of attributes, had it been performed following the decision.

Note, however, that these results can also be accounted for, using an intuition/affective hypothesis. Accordingly, participants make use of a mixture of intuitive/affect and deliberation strategies in immediate decisions, and these strategy differences are amplified as a result of the distraction/think-carefully manipulation; thinkcarefully participants being more likely to rely on remembered attributes, while distraction participants more likely to rely on the integrated values (intuition as a process of implicit integration of values). The intuitive/affective hypothesis can also be tested with manipulations that do not require a post-acquisition distraction/ think-aloud manipulation, but rather via mind-set manipulations in immediate decision-making. In our second experiment, we set out to further examine the effect of mind-set on complex multiattribute decision-making, using an affect manipulation that was designed to trigger a distinction between the intuitive/experiential and the rational/analytic modes of decision-making.

\section{EXPERIMENT 2: AFFECTIVE VS. ANALYTICAL MIND-SETS}

The second experiment presented participants with an immediate decision (without time pressure) between multi-attribute alternatives (cars) using the same materials previously employed by Dijksterhuis et al. (2006). The key difference is that the distraction/ think-carefully manipulation was replaced by a mind-set manipula- tion (based on Epstein's CEST theory; Epstein, 1994), previously used to trigger an analytic/deliberation vs. experiential/intuitive mind-set (Krauss et al., 2004). It is predicted that with such a mindset manipulation, which centers on the distinction between rulebased and emotional/affective processing, differences in decision quality in favor of the intuitive group will be obtained even in the immediate decisions.

\section{METHOD \\ Participants}

Thirty-six participants (19 female; age-range: 20-30) from TAU took part in this experiment. They were randomly assigned to the affect and analytic groups. Each group consisted of $N=18$ participants. The TAU student participants, recruited by the experimenters, volunteered to take part in a study of decision-making prior to being exposed to any decision-making lectures.

\section{Materials}

The decision materials were the same as reported in Dijksterhuis et al. (2006). They consisted of four fictitious car alternatives each composed of the same 12 binary (good/bad) attributes. One alternative (A) was composed of $66 \%$ positive attributes; two alternatives contained $50 \%$ positive attributes while one of them (D) had only $33 \%$ positive attributes.

\section{Procedure}

The first stage of the experiment consisted of the mind-set manipulation, which took $3 \mathrm{~min}$. The participants in the analytic group received a sheet of mathematical queries (e.g., multiplications; additions; subtractions; raising to power-two; and fractions) with instructions to calculate the results. The participants were told to calculate as many of the queries that they could within the 3-min interval, and were allowed to skip questions. They were also provided with a pen and pad of paper to complete the calculations. Participants in the experiential/affective group were given a task that required them to express their current emotional state (Krauss et al., 2004). Specifically, participants were told that "feelings and attitudes can be expressed through a number of measures including creative expression. We would like you to draw a picture that describes your gut-level feelings about your emotional state right now." This manipulation is motivated by the association of the intuitive system with affect (Damasio, 1994; Epstein, 1994). Participants were given a pack of colored crayons to draw with for an interval of 3 min. Participants in both groups, were told that the task will not be evaluated and, if they prefer, they can take the math/drawing sheet with them. This was done to minimize any stress that could be triggered by the mind-set manipulation.

In the second stage, all the participants were informed that they would be presented with information about four cars, which a hypothetical person views in order to choose a new car. Then the 48 pieces of information were presented (random order, not blocked) at a pace of $8 \mathrm{~s}$ per item (car and attribute combination). In the final stage the participants were asked to evaluate each of the four cars and indicate their preference for each, by ticking a location on four horizontal lines (one for each alternative), presented to them on paper. Previous research has indicated that this kind of 
(analog) response mode is more sensitive than numeric evaluations for tapping into an individual's intuitive processes (Betsch et al., 2001). Two dependent measures were examined: the "choice" fraction for the best-alternative (defined by the option that received the highest rating) and the differentiation scores (A-D).

\section{RESULTS}

\section{Manipulations}

All the participants were able to carry out the mind-set manipulations without difficulty. In particular, all participants showed high motivation and good compliance with the mode of thought manipulation (drawing or making calculations; none of them reported difficulties).

\section{Choice fraction}

The percent of participants "choosing" each option, in each of the two groups is reported in Table 3 for a subset of the participants $(N=12$ participants in the affect group and $N=13$ participants in the analytic group) ${ }^{1}$.

The percent of participants who gave option-A (the "good car") the highest rank compared to the other cars, was higher for the intuition group $(83 \%)$ when compared with the analytic group $\left(30 \% ; \chi^{2}(1)=7, p<0.01\right)$.

\section{Differentiation}

The differentiation scores were higher in the affect group (Mean $=22.5, \mathrm{SD}=10.0)$ when compared with the analytic group (Mean $=12.2 ; \mathrm{SD}=14.7)$. The difference was significant $[t(35)=2.47 ; p=0.02 ;$ two-tailed $]$.

\section{DISCUSSION}

The results of this experiment demonstrate that when participants were induced into an affective mind-set, by drawing a picture that corresponded to their current emotional state, they performed better on value integration across attributes than participants induced into a rational/analytic mind-set (as a result of solving math problems). One potential criticism to this conclusion is that this difference is the result of fatigue, triggered by the pre-decision task, higher for the math-calculations, than for the drawing. We believe this to be unlikely, as the performance in the math task was de-emphasized (participants were told they can take the drawing/ math sheet with them). However, in order to verify that the result is not due to a fatigue artifact we ran a control test, in which the same mind-set manipulation was done before a task that is not decision-related - the reading-span (Daneman and Carepenter, 1980)

${ }^{1}$ This is due to the fact that the response forms of the few participants were mistakenly lost, after having registered the differentiation score, which was the initial focus of the research.

Table 3 | Number of participants that selected each option, in the two groups.

\begin{tabular}{lllll}
\hline Group/alternative & A & B & C & D \\
\hline Analytic group & 4 & 4 & 3 & 2 \\
Intuitive group & 10 & 0 & 1 & 1
\end{tabular}

- which measures the capacity of working memory. Two groups of 10 (two females) participants were tested. The results indicate no effect of our mind-set manipulation on reading-span scores. The average scores were $2.9(\mathrm{SD}=0.54)$ for the math-group and 3 $(\mathrm{SD}=0.77)$ for the drawing group $(p>0.75)$. As the reading-span measures the working memory capacity, this makes an interpretation based on a generalized fatigue effect less likely and supports the interpretation that the mind-set effect is due to the advantage of the affective over the analytic mind-set in complex decisions.

This result supports the hypothesis that the affective/intuitive system is superior in value integration, compared with the deliberation, rule-based system, and is not subject to criticisms that have been raised against the unconscious thought effect, such as, that it reflects interference in the think-carefully condition (rather than better processing for the unconscious decision). The aim of the next experiment is to use a different manipulation that is associated with the intuitive-affective system (Epstein, 1994). While in Experiment 2 the manipulation involved the engagement of an affective/emotional mode of thought implicitly (via an unrelated task), in Experiment 3 we used an explicit manipulation (via instructions) to induce an intuitive mode of thought (Epstein, 1994; Lieberman, 2000; Ferreira et al., 2006). If intuition-instructions can make participants tap into such processes one should also expect an effect on decision quality in immediate decisions, as suggested by studies, in which participants were asked to reflect on, or justify the reason for their preference, showing that deliberation on reasons can bias decision-making (Wilson and Schooler, 1991; Wilson et al., 1993, 1995; Levine et al., 1996).

A second aim in the next two experiments was to address the ecological criticism often raised against UTT, which points out that in many of these studies, participants are presented with very fragmented information (48 pieces of information in a randomized order) and have no further access to that information during the deliberation stage. It can then be argued that such situations are hardly representative (and of little relevance) to real-life decisions (Shanks, 2006). In our next two experiments we attempted to modify the mode in which the information is presented so that it resembled more real-life choice situations. In both experiments, the main manipulation involved deliberation vs. mind-set instructions.

\section{EXPERIMENT 3: INTUITIVE VS. ANALYTIC MIND-SETS}

A common situation in complex decision-making is that one examines different aspects of the information about a choice several times. Consider, for example, a person who struggles to decide which of three available apartments to purchase. It would be likely that the person would visit the three apartments a few times, each time paying more attention to other aspects, and that this information will then be integrated toward a decision. Furthermore, the information is unlikely to be fragmented, as was the case in our previous two experiments. To mimic such a situation, in these two experiments we presented the information about choosing a flatmate (a topic that is ecological for most of our participants who are undergraduate students) in blocks of four attributes (each block is analogous to a visit to one apartment). This means that the participants are shown a slide with four attributes of flatmate A for $12 \mathrm{~s}$, before being shown a slide with the same four attributes for flatmate B (again $12 \mathrm{~s}$ ) and then 


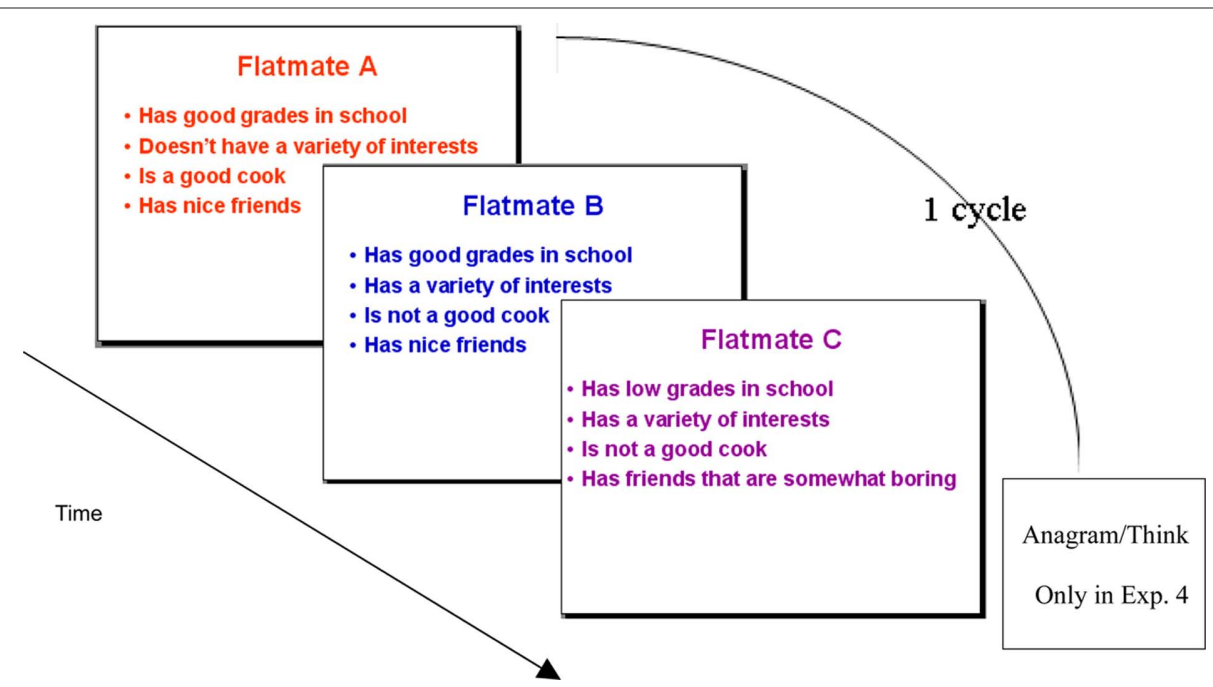

FIGURE 2 | Presentation of the choice material in Experiments 3 and 4: the first of three cycles, in which the information about the flatmates is presented. The information is presented, with a sequence of slides, each slide containing four attributes about one flatmate and is shown for $12 \mathrm{~s}$. Each cycle starts with four attributes of flatmate A, followed by four attributes of flatmate B, and last four attributes of flatmate $\mathrm{C}$; the fourth box, Anagram/Think, only applies to Experiment 4. Three cycles of slides are presented, corresponding to a total of 12 attributes for each flatmate. for flatmate C (Figure 2). Following this cycle, two new cycles were presented, with a new set of four attributes each, which sum to a sequence of nine slides, with a total of 12 attributes for each of the three flatmates. We reasoned that faced with this type of presentation, participants will have the choice of focusing their decision on value integration within an alternative (a holistic decision) or on comparisons of attributes between alternatives (a featural decision; Lerouge, 2009); which they can do on the basis of information in working memory. Instead of the drawing/ emotional manipulation, here we used a more direct manipulation. Participants were instructed to rely either on their intuition (gut-feeling) or on their reasons (which they may need to explain). Based on our previous experiment and the association between system-I with both affective processing and intuition (Epstein, 1994), as well as on the basis of previous studies that demonstrated negative effects of over-thinking on the quality of preference (Wilson and Schooler, 1991; Wilson et al., 1993) we predict that the choice and the differentiation scores will be higher for the intuitive group, who is more likely to evaluate the flatmates holistically (i.e., integrate across attributes) rather than on the basis of comparisons of attributes from working memory.

\section{METHOD \\ Participants}

Twenty-four participants (11 female; age-range 21-41) took part in Experiment 3. Most were students at Birkbeck or UCL and were paid $5 \mathfrak{E}$ for participation in the experiment. The participants were randomly assigned to the two mind-set conditions (12 to each).

\section{Materials}

The choice alternatives consisted of three flatmates, which were composed of a set of 12 binary attributes (sense of humor, tidiness, etc.; see Appendix). Pilot testing was used to exclude attributes that were too important and would therefore have a disproportionate impact (in comparison to the other attributes) on the decision. One of the flatmates (the best option, A) was composed of $66 \%$ positive attributes ( 8 out of 12), the second flatmate (B) was composed of $50 \%$ positive attributes while the third flatmate $(\mathrm{C})$ was composed of $33 \%$ positive attributes. A second pilot on 17 participants (asked to give weights to the attributes, in the context of choosing a flatmate, on a scale of 1-9) confirmed that the weighted utility was highest for $\mathrm{A}($ Mean $=50.5, \mathrm{SD}=7.9)$, middle for $\mathrm{B}($ Mean $=39.4$; $\mathrm{SD}=6.3)$ and lowest for $\mathrm{C}($ Mean $=25.3 ; \mathrm{SD}=4.0)$; all differences are significant $(p s<0.001)$.

\section{Procedure}

Participants were first given instructions to induce intuitive or analytic mind-sets (see Ferreira et al., 2006, Experiment 1 for a similar manipulation). Participants in the intuitive conditions were told that "Research has shown that the best decisions are the ones made using intuition" and were encouraged to base their evaluation on their "GUT-FEELING" and to make a distinction between the overall attractiveness of each flatmate. They were told that they will be given as much time as needed to make an evaluation, but warned that thinking too long does not help (thus better to use gutfeeling). Participants in the analytical group were told that "Research has shown that the best decisions are the ones made using logic and rational thought" and were encouraged to think-carefully and logically about the three flatmates and about the reasons to prefer one flatmate to another one (see Appendix for detailed instructions). Following the mind-set instruction the information about the three flatmates was presented (Figure 2).

In order to facilitate the participant's ability to bind the attributes of each flatmate and differentiate them from that of the other flatmates, the attributes of each flatmate were shown in a different color. The order of the alternatives in a cycle was always, $\mathrm{A} / \mathrm{B} / \mathrm{C}$, 
however, the relation between the good/middle/bad and A/B/C was counterbalanced. Finally, the participants were required to enter a rating (on a scale 1-10) for each of the flatmates.

\section{RESULTS AND DISCUSSION}

There were no significant differences in the fraction of participants who rated the good alternative highest in the two conditions (7/12 in both). However, the differentiation scores (A-C), were significantly larger than zero, only for the intuitive group $[$ Mean $=2.42 ; \mathrm{SD}=2.47 ; t(11)=3.4, p<0.01]$. The differentiation scores of the analytic group were not significantly different from zero [Mean $=0.58 ; \mathrm{SD}=2.84 ; t(11)=0.71, p=0.58$ ], and they were smaller than for the intuitive group $[t(22)=1.69$; $p<0.05$; one-tail]. Although only significant at a one-tail level, this result is consistent with our prediction of an advantage in favor of the intuitive mind-set group (see also Wilson and Schooler, 1991; Wilson et al., 1993, 1995). The relatively small effect is due to the large individual differences, suggesting that the instruction manipulations may not have been effective enough for all participants.

The aim of the fourth experiment was to strengthen the manipulation, and at the same time to increase the ecological validity to that of real-life decisions. To do this, we combined the mind-set manipulation with a distractor task (Lerouge, 2009). The aim of was to test if combining the two types of manipulation in an integrated task results in enhanced decision quality.

\section{EXPERIMENT 4: DISTRACTION AND INTUITION TOGETHER}

Experiment 4 was identical to Experiment 3, except that a distraction/think manipulation was added in combination with the mind-set instruction (Figure 2). This manipulation was chosen so as to maximize the difference between the intuitive vs. analytic modes of thought. For the participants in the analytic condition, this manipulation involved analyzing the reasons for liking or disliking each of the three flatmates. For the participants in the intuitive condition, the manipulation involved solving anagrams. Note that the aim was not to carry out a $2 \times 2$ examination of instructions with distraction-type, but rather to contrasts the extreme modes of thought: intuitive vs. conscious deliberation, under the assumption that while analyzing would strengthen the analytic instruction mind-set, the anagrams will strengthen the intuitive mind-set. Furthermore, the design differs from the standard think-aloud/anagram paradigm (Dijksterhuis et al., 2006), in which the manipulation is only applied at the end of the information acquisition (when some of the participants may already have made a decision). Here the manipulation was inter-spaced with the acquisition itself. Instead of $3 \mathrm{~min}$ at the end, it includes three intervals of about $1 \mathrm{~min}$, after each cycle of presentation (see Figure 2).

There are few additional motivations for inter-spacing the acquisition and think/distraction intervals. First, we believe that this procedure is more similar to real-life decision situations, in which the acquisition of information and the decision time are proceeding in parallel, allowing the participants a better opportunity to make use of the information (not yet forgotten) during the think/distraction intervals. Second, this procedure reduces the likelihood that participants decide before they are exposed to the manipulation, which may often happen in the standard unconscious thought experiments. Finally, while the inter-spacing of think-intervals during the acquisition of decision information, might trigger anchoring and adjustment mechanisms, resulting in less holistic reasoning (Pennington and Hastie, 1992), the interspacing of anagram reasoning, could facilitate holistic processing and integration.

\section{METHOD \\ Participants}

Twenty-nine participants from the Interdisciplinary center (IDC) Herzliya (23 female; age-range 20-30) took part in Experiment 4. They were randomly assigned to the two mind-set conditions and received credit for their participation.

\section{Materials}

Same as for Experiment 3.

\section{Procedure}

The mind-set instructions and the presentation of the information were the same as in Experiment 3 (see Appendix), the only difference being the time windows (every cycle of 12 items; see Figure 2), in which the participants are asked to reason about the flatmate (analytic group) or to solve anagrams (intuitive group). The reasoning (analytic group), was prompted by presenting three queries asking for reasons for liking/disliking each flatmate; participants could proceed to the next query (or next stage in the experiment) only when they typed a response. They were allowed to type "don't know" if they could not answer (those answers were coded as "incorrect"). This procedure was repeated at the end of each presentation cycle and was not time limited. The intuitive group were asked to solve three anagrams, following each three-slide presentation cycle. The anagram task was not time limited. Each anagram was presented on the screen until the participant typed a response ("don't know responses were allowed and were coded as "incorrect"). Finally, as in Experiment 3 , the participants were required to enter a rating (on a scale 1-10) for each of the flatmates.

\section{RESULTS}

\section{Anagram and Reasoning tasks}

In both manipulations the participants showed a high accuracy in their ability to answer queries about the flatmates (96\%) and in their ability to solve the anagrams (89\%). The average time that participants in the analytical condition took to answer each query about liking or disliking the flatmates was $22.5 \mathrm{~s}(\mathrm{SD}=15.4)$, i.e., about $1 \mathrm{~min}$ per cycle. The average time that participants in the intuitive condition took to solve each anagram was $22.7(\mathrm{SD}=39)$, i.e., also about $1 \mathrm{~min}$ per cycle.

\section{Decision Quality}

There was a significant difference in the fraction of participants who gave the good alternative, $\mathrm{A}$, the highest rating in the two conditions (15/15 in the intuitive condition, 9/14 in the analytical condition). This difference reached significance $\left[\chi^{2}(1)=6.47 ; p=0.01\right]$. There was also a significant difference in the differentiation scores between the two groups in 
favor of the intuitive-distraction group $($ Mean $=2.8, \mathrm{SD}=1.74$ vs. Mean $=1.57, \mathrm{SD}=1.22$ ). This difference was significant $[t(27)=2.18, p<0.05]$.

\section{DISCUSSION}

The results of Experiment 4 appear to have succeeded in strengthening the manipulation by combining the intuitive/analytic instructions with a distraction/think manipulation. This resulted in a significant difference in both the choice and the differentiation scores. As the two Experiments ( 3 and 4) were performed on different participants (London vs Tel-Aviv, though they were both with undergraduate students and the same materials were used), further research is needed to establish whether the distraction manipulation contributes to the intuitive advantage beyond what is obtained with intuitive/analytic instructions alone. The important result, however, is that in both experiments an advantage in favor of the intuitive mode of thought was demonstrated in a complex decision-making task that attempted to maintain aspects of ecological validity. These results are consistent both with the UTT, according to which incubation processes take place during the solution of the anagrams, and with the intuitive-affective hypothesis, according to which both the instructions and the anagram distraction push participants to rely more on intuitive mind-sets.

One additional point is important to make in relation to Experiment 4. The results of this experiment are remarkable given that the participants in the analytic-deliberation group had actually more useful time to deliberate on their decisions compared to the participants in the intuitive-anagram group. Unlike in the previous studies with the unconscious thought paradigm, which has been criticized for placing the participants in the think-carefully condition in a situation that is not helpful for decision-making (because most of the information is forgotten by the time they are given time to think), here the "thinking" intervals are introduced during the presentation of the material in a way that one may expect to help them integrate the information. The fact that the same intervals, filled with anagram activity, had a larger effect on the differentiation scores, provides some additional support to the UTT and suggests that it may have more ecological relevance than previously thought. This is consistent with the suggestion that unconscious thought during distraction intervals can play a role in organizing the information in the unconscious thought experiments (Strick et al., 2010).

\section{GENERAL DISCUSSION}

The four experiments reported here provide support for the claim that intuitive-affective strategies can outperform deliberation/analytic strategies in value integration, an operation that is critical for complex decision-making. To summarize, in Experiment 1 we obtained a replication of the unconscious thought effect (in both choice and differentiation scores) using a distraction manipulation (Dijksterhuis, 2004; Dijksterhuis, et al., 2006) with alternatives that were constructed, for each participant, with a definite order of subjective quality. In Experiment 2 and 3 we used a mind-set manipulation (implicit-affective in Experiment 2 and explicit-intuition in Experiment 3) without a distraction. The results were in favor of the groups which were induced into affective or intuitive mind-sets compared with the groups induced into deliberation-analytic mind-sets. Finally, in Experiment 4, we combined the mind-set (intuitive-analytic) and the distraction manipulation into an integral task. Here the distraction and the think-intervals were interspersed with the presentation of the material, allowing the participants a better opportunity to integrate values or to recollect them. The results favored the intuitive-distraction group compared with the analytic-deliberation group.

Given the fact that some of the criticism against the UTT is based on failed replications (Acker, 2008, but see Strick et al., unpublished manuscript ${ }^{2}$ ), the results of our first experiment provide some support for the UTT theory (Boss et al., 2008; Dijksterhuis and Nordgren, 2006). Nevertheless, we believe that further research is needed in order to decide whether unconscious thought during a distractor interval is the critical process that contributes to the unconscious thought, and it is not our aim to evaluate this position here. We believe that one should keep an open mind toward this possibility, which is supported by some independent research on incubation in insight problems (Zhong et al., 2008), but which faces a number serious challenges. First, as recently shown by Payne et al. (2008), participants in a think-condition can outperform participants in distractor conditions, if the interval of the thinkperiod is self-paced rather than fixed ${ }^{3}$. Second, the fact that many studies have failed to replicate the results, indicate that the effect is highly sensitive to experimental conditions (e.g., instructions) and to individual differences (Lassiter et al., 2009; but see Strick et al., 2010). Part of the difficulty may be related to the nature of the distraction/think paradigm, which is such that the conscious/ unconscious manipulation is applied only after the participants have been exposed to the choice material, by which time participants could already have formed a decision.

Unlike UTT, which makes a somewhat stronger claim and has attracted considerable debate, the view that the intuitive-affective system is critical for fast value integration is consistent with the wider literature that includes neuropsychological (Damasio, 1994; Lieberman, 2000; Bechara and Damasio, 2005; Fellows 2006; Weller et al., 2007) and experimental methods (Wilson and Schooler, 1991; Wilson et al., 1993, 1995; Betsch, et al., 2001; Bierman et al., 2005). According to this view, distraction is only one type of manipulation, which together (or in combination) with mind-set can enhance decision quality by making participants use affective-intuitive strategies. Results consistent with this hypothesis were also obtained by Wilson and colleagues using a different experimental paradigm with an indirect measure of decision quality (Wilson and Schooler, 1991; Wilson et al., 1993). For example, Wilson and Schooler (1991) found that participants who analyzed their reasons for liking different strawberry jams made preference decisions that corresponded less well with those of experts than did those of participants who did not analyze the reasons for their attitudes. In another experiment (Wilson et al., 1993), people who did, or did not, analyze the reasons

${ }^{2}$ www.unconsciouslab.com/publications/Paper_Meta.doc

${ }^{3}$ The experiment, however, was carried out with alternatives characterized by numerical values, and it remains to be seen if this effect persists with qualitative attributes that may be more natural for the intuitive or unconscious thought system (Dijksterhuis and Nordgren, 2006; Dijksterhuis et al., 2009). 
why they liked five different posters were given an opportunity to select one of the posters to take home. When they were contacted a few weeks later, reasoners were less satisfied with their choices than were non-reasoners.

The hypothesis that affect and intuition mind-sets can enhance decision quality is consistent with the two-system theory (Epstein, 1994; Sloman, 1996; Evans, 2003, 2008; Kahneman, 2003; Ferreira et al., 2006; Kahneman and Frederick, 2007) which has recently been subject to vigorous criticism (Keren and Schul, 2009). In the following we briefly address this challenge and then we discuss a number of implications for two issues of broad general interest. The first one is conceptual and it involves the complimentary roles of the rational and the intuitive systems in decision-making. The second involves implications for enhancing decision-making in real-life situations.

The essence of the Keren and Schul criticism of the two-system theory can be captured by the following objections: (i) lack of consistency and clarity on what the properties of the systems are (different authors use different properties); (ii) are the properties of the systems well aligned in all tasks?; (iii) are the systems isolable?; and (iv) can the evidence used to support the theory be accounted for by shifting decision criteria within a single cognitive system? As the authors state, the criticism is not against a two process (or mode of thought) approach, nor is it a plea for the rejection of the two-system theory, but rather it is a request for clarity (p. 547). We believe this is an important challenge, which we wish to address.

The view we favor (Damasio, 1994; Lieberman, 2000; Ferreira et al., 2006) is a milder version of the two-system theory, which sees the systems as interacting rather than as totally isolated during normal decision-making. We believe there is strong converging evidence to suggest that there are different brain mechanisms that specialize in the affective integration or implicit learning of this type of information resulting in affective value and mediated by dopamine (system-1), as opposed to brain mechanisms (system-2) that specialize in sequential analysis of decision aspects (Lieberman, 2000; Greene et al., 2001; Lieberman et al., 2004; Bechara and Damasio, 2005; De Martino et al., 2006; for review see, Weber and Johnson, 2009). While these two mechanisms interact in normal decision-making, each of them can in principle be used to make decisions, though (as patients studies show) not very efficient ones. Furthermore, there is no reason to exclude additional systems (or subsystems) that have some mixed properties: e.g., spreading activation processes in the semantic system, which share the parallel type of process with the first system but not its affective value based processing ${ }^{4}$. Nevertheless, we believe that it is meaningful (and coherent) to focus on the following main properties and functions of the two extreme systems: system-1 (intuition) is parallel, extracts gist (holistic), and results in affective states, which are open to phenomenological awareness (Block, 2007) in their end result but not in their operation (or stages). While, in contrast, system-2 (deliberation) is sequential, rule-based (e.g., lexicographic), and has access to the stages of processing.

${ }^{4}$ This is, in fact consistent, with the two-system theory as presented by Kahneman (2003), which envisages of the intuition system to share properties with both the perceptual system and the controlled cognitive system.
Lets us illustrate this for the type of decisions discussed here. When presented with few alternatives, each characterized by many attributes, system-2 would play the role of sequentially inspecting attributes and relations (this car is more economical than the other), while system-1 has the function of generating an affective integration of the values. A model based on this idea, was recently presented by Glöckner and Betsch (2008b), who have further developed the coherency and constraint-satisfaction theories of decision-making (Thagard, 1989; Holyoak and Simon, 1999). According to Glöckner and Betsch (2008b), system-2 has the role of searching for information relevant to the decision, when the present information does not allow the intuitive system (characterized as a parallel constraint-satisfaction network) to reach a decision with enough confidence. This model, illustrates the idea that optimal decisions require a coordinated interaction of the two-systems: repeated examination of the material (or deliberation on the various features) allows the intuitive system a better opportunity to integrate (learning increases with the number of presentations) ${ }^{5}$. This learning process, however, has limitations. The potential downside of an excessive reliance on the deliberation system is that one may get trapped into loops: A is better because of reason-1, but B is better because of reason-2, and so on. This can then lead to a reliance on conscious lexicographic heuristics that totally bypass the intuitive system and its ability to integrate values explaining effects of the type reported here and in other studies. These studies demonstrated that excessive deliberation (on reasons) distorts decisions (Wilson and Schooler, 1991; Wilson et al., 1995), as well as the decision deficits of patients with lesions in the ventromedial prefrontal cortex (Bechara et al., 1997; Bechara and Damasio, 2005).

The two system theory has implications for how to enhance decision-making in real-life situations. It suggests that, after having deliberated about a decision for as long as one feels comfortable, it does make sense to put it aside, or as suggested by Dijsksterhuis, to "sleep on it," in order to allow ourselves the opportunity to tap into the integrated value computed by the intuitive/affective (or unconscious) system. We believe that, independent of the theoretical explanation (unconscious thought or intuition) our results (in particular Experiment 4) provide evidence for this conclusion, although at this stage, it should be taken with caution. There are surely factors that can bias the affective system in ways irrelevant to the decision situation (Winkielman et al., 1997; Raghunathan and Pham, 1999; Finucane et al., 2000; Greene et al., 2001; Au et al., 2003), so that the optimal decision strategy is likely to depend on a variety of contingencies. Future work that examines objective measures of decision quality in reallife problems, in which attributes are not explicit (e.g., Dijksterhuis et al., 2009), and which takes into account individual differences, are important to better understand how to facilitate decision-making.

\section{ACKNOWLEDGMENTS}

We wish to thank Jyoti Palha for preparing and testing participants in Experiment 1, Eldad Shochat and Efrat Eshar for their part in preparing materials and testing in Experiment 2, Henk Haarmann and Andrei Teodorecu for a critical reading and helpful discussions, to Jonathan Baron for comments on a previous version of the manuscript and David Shanks for suggesting the need for an affect mind-set manipulation.

${ }^{5}$ If in addition, we assume that deliberation system will inspect more often features that are more important, this could contribute to subjective weighting. 


\section{REFERENCES}

Acker, F. (2008). New findings on unconscious versus conscious thought in decision making: additional empirical data and meta-analysis. Judgm. Decis. Mak. 3, 292-303.

$\mathrm{Au}, \mathrm{K}$., Chan, F., Wang, D., and Vertinsky, I. (2003). Mood in foreign exchange trading: cognitive processes and performance. Organ. Behav. Hum. Decis. Process 91, 322-338.

Bechara, A., and Damasio, A. R. (2005). The somatic marker hypothesis: a neural theory of economic decision. Games Econ. Behav. 52, 336-372.

Bechara, A., Damasio, A. R., Damasio, H., and Anderson, S. W. (1994). Insensitivity to future consequences following damage to human prefrontal cortex. Cognition 50, 7-15.

Bechara, A., Damasio, H., Tranel, D., and Damasio,A.R. (1997). Deciding advantageously before knowing the advantageous strategy. Science 275, 1293-1295.

Betsch, T., Plessner, H., Schwieren, C., and Gütig, R. (2001). I like it but I don't know why: a value-account approach to implicit attitude formation. Pers. Soc. Psychol. Bull. 27, 242-253.

Bierman, D., Destrebecqz, A., and Cleeremans, A. (2005). Intuitive decision making in complex situations: somatic markers in an implicit artificial grammar learning task. Cogn. Affect. Behav. Neurosci. 5, 297-305.

Block, N. (2007). Consciousness, accessibility, and the mesh between psychology and neuroscience. Behav. Brain Sci. 30, 481-499.

Bos, M. W., Dijksterhuis, A., and van Baaren, R. B. (2008). On the goaldependency of unconscious thought. J. Exp. Soc. Psychol. 44, 1114-1120.

Calvillo, D. P., and Penaloza, A. (2009). Are complex decisions better left to the unconscious? Further failed replications of the deliberation-withoutattention effect. Judgm. Decis. Mak. 4, 509-517.

Damasio, A. R. (1994). Descartes' Error: Emotion, Reason, and the Human Brain. New York: Putnam.

Daneman, M., and Carpenter, P.A. (1980). Individual differences in working memory and reading. J. Verbal Learn. Verbal Behav. 19, 450-466.

Dawes, R. M., and Corrigan, B. (1974). Linear models in decision making. Psychol. Bull. 81, 95-106.

De Martino, B., Kumaran, O., Seymour, B., and Dolan, R. (2006). Frames, biases, and rational decision-making in the human brain. Science 313, 684-687.

Dijksterhuis, A. (2004). Think different: the merits of unconscious thought in preference development and decision making. J. Pers. Soc. Psychol. 87, 586-598.
Dijksterhuis, A., Bos, M. W., Nordgren, L. F., and van Baaren, R. B. (2006). On making the right choice: the deliberation without attention effect. Science 311, 1005-1007.

Dijksterhuis, A., Bos, M. W., van der Leij, A., and van Baaren, R. B. (2009). Predicting soccer matches after unconscious and conscious thought as a function of expertise. Psychol. Sci. 20, 1381-1387.

Dijksterhuis,A., and Nordgren,L.F. (2006). A theory of unconscious thought. Perspect. Psychol. Sci. 1, 95-109.

Edwards, W., and Fasolo, B. (2001). Decision technology. Annu. Rev. Psychol. 52, 581-606.

Edwards, W., and Newman, J. R. (2003). "Multiattribute evaluation," in Judgment and Decision Making: An Interdisciplinary Reader, eds H. R. Arkes and K. R. Hammond (New York: Cambridge University Press), 13-37.

Epstein, S. (1994). Integration of the cognitive and the psychodynamic unconscious. Am. Psychol. 49, 709-724.

Evans, J. S. B. T. (2003). In two minds: dual process accounts of reasoning. Trends Cogn. Sci. 7, 454-459.

Evans, J. S. B. T. (2008). Dual-processing accounts of reasoning, judgment, and social cognition. Annu. Rev. Psychol. 59, 255-278.

Fellows, L. K. (2006). Deciding how to decide: ventromedial frontal lobe damage affects information acquisition in multi-attribute decision making. Brain 129, 944-952.

Ferreira, M. B., Garcia-Marques, L., Sherman, S. J., and Sherman, J. W. (2006). Automatic and controlled components of judgment and decision making. J. Pers. Soc. Psychol. 91, 797-813.

Finucane, M. L., Alhakami, A., Slovic, P., and Johnson, S. M. (2000). The affect heuristic in judgments of risks and benefits. J. Behav. Decis. Mak. 13, 1-17.

Franklin, B. (1772). The Writings of Benjamin Franklin, Vol. 5, Albert Henry Smyth, 1906. New York: The Macmillan Company.

Gigerenzer, G., and Goldstein, D. G. (1996). Reasoning the fast and frugal way: models of bounded rationality. Psychol. Rev. 103, 650-669.

Glöckner, A. (2008). "Does intuition beat fast and frugal heuristics? A systematic empirical analysis," in Intuition in Judgment and Decision Making (Mahwah, NJ: Lawrence Erlbaum Associates Publishers), 309-325, xix, 335.

Glöckner, A., and Betsch, T. (2008a). Multiple-reason decision making based on automatic processing. J. Exp. Psychol. Learn. Mem. Cogn. 34, 1055-1075.
Glöckner, A., and Betsch, T. (2008b). Modeling option and strategy choices with connectionist networks: towards an integrative model of automatic and deliberate decision making. Judgm. Decis. Mak. 3, 215-228.

Gonzalez-Vallejo, C., Lassiter, G. D., Bellezza, F. S., and Lindberg, M. J. (2008). "Save angels perhaps:" a critical examination of unconscious thought theory and the deliberation without attention effect. Rev. Gen. Psychol. 12, 282-296.

Greene, J. D., Sommerville, R. B., Nystrom L. E., Darley, J. M., and Cohen, J. D. (2001).An fMRI investigation of emotional engagement in moral judgment. Science 293, 2105-2108.

Ham, J., and Van den Bos, K. (2010). The merits of unconscious processing of directly and indirectly obtained information about social justice. Soc. Cogn. 28, 180-190.

Ham, J., van den Bos, K., and Van Doorn, E. (2009). Lady Justice thinks unconsciously: unconscious thought can lead to more accurate justice judgments. Soc. Cogn. 27, 509-521.

Holyoak, K. J., and Simon, D. (1999). Bidirectional reasoning in decision making by constraint satisfaction. $J$. Exp. Psychol. Gen. 128, 3-31.

Igou, E. R., and Bless, H. (2007). On undesirable consequences of thinking: framing effects as a function of substantive processing. J. Behav. Decis. Mak. 20, 125-142.

Kahneman, D. (2003). A perspective on judgment and choice: mapping bounded rationality. Am. Psychol. 58, 697-720.

Kahneman, D., and Frederick, S. (2007) Frames and brains: elicitation and control of response tendencies. Trends Cogn. Sci. (Regul. Ed.) 11, 45-46.

Keeney, R. L., and Raiffa, H. (1976). Decisions with Multiple Objectives: Preference and Value Tradeoffs. New York: Wiley.

Keren, G., and Schul, Y. (2009). Two is not always better than one: a critical evaluation of two-system theories. Perspect. Psychol. Sci. 4, 533-550.

Knowlton, B. J., Squire, L. R., Paulsen, J. S., Swerdlow, N. R., Swenson, M., and Butters, N. (1996). Dissociations within nondeclarative memory in $\mathrm{HD}$. Neuropsychology 10, 538-548.

Krauss, D. A., Lieberman, J. D., and Olson, J. (2004). The effects of rational and experiential information processing of expert testimony in death penalty cases. Behav. Sci. Law 22, 801-822.

Lassiter, G. D., Lindberg, M. J., Gonza'lezVallejo, C., Bellezza, F. S., and Phillips, N. D. (2009). The deliberation without-attention effect: evidence for an artifactual interpretation. Psychol. Sci. 20, 671-675.

Lerouge, D. (2009). Evaluating the benefits of distraction on product evaluations: the mind-set effect. J. Consum. Res.36, 367-379.

Levine, G. M., Halberstadt, J. B., and Goldstone, R. L. (1996). Reasoning and the weighing of attributes in attitude judgments. J. Pers. Soc. Psychol. 70, 230-240.

Lieberman, M. D. (2000). Intuition: a social cognitive neuroscience approach. Psychol. Bull. 126, 109-137.

Lieberman, M. D., Jarcho, J. M., and Satpute, A. B. (2004). Evidence-based self-knowledge: an fMRI study. J. Pers. Soc. Psychol. 87, 421-435.

Newell, B. R., Wong, K. Y., Cheung, J. C. H., and Rakow, T. (2009). Think, blink, or sleep on it? The impact of modes of thought on complex decision making. Q. J. Exp. Psychol. 62, 707-732.

Payne, J. W., Samper, A., Bettman, J. R., and Luce, M. F. (2008). Boundary conditions on unconscious thought in complex decision making. Psychol. Sci. 19, 1118-1123.

Pennington N., and Hastie, R. (1992). Explaining the evidence: the story model of jury decision-making. J. Pers. Soc. Psychol. 62, 189-206.

Raghunathan, R., and Pham, M. T. (1999). All negative moods are not equal: motivational influences of anxiety and sadness on decision making. Organ. Behav. Hum. Decis. Process. 79, 56-77.

Reber, A. S. (1993). Implicit Learning and Tacit Knowledge: An Essay on the Cognitive Unconscious. New York: Oxford University Press.

Rey, A., Goldstein, R. M., and Perruchet, P. (2009). Does unconscious thought improve complex decision making? Psychol. Res. 73, 372-379.

Savage, L. J. (1954). The Foundations of Statistics, 1972 Edn. New York: Dover.

Shanks, D. R. (2006). Complex choices better made unconsciously? Science 313, 760-761.

Simon, H. A. (1955). A behavioral model of rational choice. Q. J. Econ. 69, 99-118.

Sloman, S. A. (1996). The empirical case for two systems of reasoning. Psychol. Bull. 119, 3-22.

Stout, J. C., Busemeyer, J. R., Lin, A., Grant, S. J., and Bonson, K. R. (2004). Cognitive modeling analysis of the decision-making processes used by cocaine abusers. Psychon. Bull. Rev. 11, 742-747.

Strick, M., Dijksterhuis, A., van Baaren, R. B., and Nordgren, L. F. (2010). Unconscious-thought effects take 
place off-line, not on-line. Psychol. Sci. Available at: http://pss.sagepub. com/content/early/2010/02/26/0956 797610363555

Thagard, P. (1989). Explanatory coherence. Behav. Brain Sci. 2,435-467.

Thorsteinson, T. J., and Withrow, S. (2009). Does unconscious thought outperform conscious thought on complex decisions? A further examination. Judgm. Decis. Mak. 4, 235-247.

Tversky,A. (1969). Intransitivity of preferences. Psychol. Rev. 76, 31-48.

Tversky, A., and Kahneman, D. (1974). Judgment under uncertainty: Heuristics and biases. Science 185, 1124-1131.

Weber, E. U., and Johnson, E. J. (2009). Mindful judgment and decision making. Annu. Rev. Psychol. 60, 53-85.

Weller, J. A., Levin, I. P., Shiv, B., and Bechara, A. (2007). Neural correlates of adaptive decision making for risky gains and losses. Psychol. Sci. 18, 958-964.

Wilson, T. D., Hodge, S. D., and LaFleur, S. J. (1995). Effects of introspecting about reasons: inferring attitude from accessible thoughts. J. Pers. Soc. Psychol. 69, 16-28.

Wilson, T. D., Lisle, D. J., Schooler, J. W., Hodges, S. D., Klaaren, K. J., and LaFleur, S. J. (1993). Introspecting about reasons can reduce post-choice satisfaction. Pers. Soc. Psychol. Bull. 19, 331-339.

Wilson, T. D., and Schooler, J. W. (1991). Thinking too much: introspection can reduce the quality of preferences and decisions. J. Pers. Soc. Psychol. 60, 181-192.

Winkielman, P., Zajonc, R. B., and Schwarz, N. (1997). Subliminal affective priming resists attributional interventions. Cogn. Emot. 11, 433-465.

Zhong, C. B., Dijksterhuis, A., and Galinsky, A. D. (2008). The merits of unconscious thought in creativity. Psychol. Sci. 19, 912-918.

Conflict of Interest Statement: The authors declare that the research was conducted in the absence of any commercial or financial relationships that could be construed as a potential conflict of interest.
Received: 09 January 2011; paper pending published: 30 January 2011; accepted: 22 February 2011; published online: 15 March 2011.

Citation: Usher M, Russo Z, Weyers M, Brauner R and Zakay D (2011) The impact of the mode of thought in complex decisions: intuitive decisions are better. Front. Psychology 2:37. doi: 10.3389/fpsyg.2011.00037

This article was submitted to Frontiers in Cognitive Science, a specialty of Frontiers in Psychology.

Copyright @ 2011 Usher, Russo, Weyers, Brauner and Zakay. This is an open-access articlesubject to an exclusive license agreement between the authors and Frontiers Media SA, which permits unrestricted use, distribution, and reproduction in any medium, provided the original authors and source are credited. 


\section{APPENDIX ATTRIBUTES FOR HOLIDAY-ALTERNATIVES (EXPERIMENT 1)}

- Good/bad whether

- Friendly/not friendly staff

- Easy/not easy access

- Variety of activities (limited)

- Nice/limited room view

- Many/few near restaurants

- Breakfast (not) included

- Many/no proximal shops

- Rooms are (not) air-conditioned

- There is (no) TV in the room

- (No) beautiful beaches

- Small/spacious rooms

See Dijksterhuis et al., 2006, for attributes of car-materials.

\section{FLATMATE MATERIAL (EXPERIMENTS 3 AND 4) Flatmate A}

- Has good grades in school

- Does not have a variety of interests

- Is a good cook

- Has nice friends

- Takes care of his/her physical appearance

- Has a good income

- Does not have similar tastes to you

- Is fun to be with

- Is a relaxed and easygoing person

- Does not have a sense of humor

- Sometimes leaves dirty dishes in the sink

- Plays pleasant music while at home

\section{Flatmate B}

- Has good grades in school

- Has a variety of interests

- Is not a good cook

- Has nice friends

- Does not take care of his/her physical appearance

- Has a good income

- Does not have similar tastes to you

- Is not fun to be with

- Is a bit uptight

- Has a sense of humor

- Sometimes leaves dirty dishes in the sink

- Plays pleasant music while at home

\section{Flatmate C}

- Has low grades in school

- Has a variety of interests

- Is not a good cook

- Has friends that are somewhat boring

- Does not take care of his/her physical appearance

- Does not have a good income

- Has similar tastes to you

- Is not fun to be with

- Is a bit uptight

- Has a sense of humor

- Does not leave dirty dishes in the sink

- Plays unpleasant music while at home

\section{EXPERIMENT 3: INSTRUCTIONS}

a) Reasoning condition

Imagine you have to find a flatmate to share an apartment in London. You will be presented with information about three hypothetical flatmates, described by various (positive/negative) attributes. You will then be asked to rate your evaluation of all three flatmates (how "good" or "bad" you feel it would be to share a flat with them) on a scale of 1-10. Research has shown that the best decisions are made using logic and rational thought. Therefore it is important that you think-carefully and logically about how much you like each flatmate. In particular, you should think about the reasons you have to prefer one flatmate to another one. You will be given as much time as you will need to make your judgment (but this will be done from memory, you cannot take notes). After you have made your decision you will be asked to justify it by giving the reasons for your choice.

(b) Intuitive condition

Imagine you have to find a flatmate to share an apartment in London. You will now be presented with information about three hypothetical flatmates, described by various (positive/negative) attributes. You will then be asked to rate your evaluation of all three flatmates (how "good" or "bad" you feel it would be to share a flat with them) on a scale of 1-10. Research has shown that the best decisions are made using intuition rather than logic. Therefore, try to base your evaluation and choice preference on your GUT-FEELING about how much you like or dislike the three flatmates, rather than trying to think logically or rationally about them. You will be given as much time as you will need to make your judgment (but this will be done from memory, you cannot take notes). Remember that you should only use your intuitive feeling. 